\title{
Energy-Efficient Forwarding Strategies for Geographic Routing in Lossy Wireless Sensor Networks.
}

\author{
Karim Seada, Marco Zuniga, Ahmed Helmy, Bhaskar Krishnamachari \\ Department of Electrical Engineering \\ University of Southern California \\ \{seada, marcozun, helmy, bkrishna\}@usc.edu
}

\begin{abstract}
Recent experimental studies have shown that wireless links in real sensor networks can be extremely unreliable, deviating to a large extent from the idealized perfect-receptionwithin-range models used in common network simulation tools. Previously proposed geographic routing protocols commonly employ a maximum-distance greedy forwarding technique that works well in ideal conditions. However, such a forwarding technique performs poorly in realistic conditions as it tends to forward packets on lossy links. We identify and illustrate this weak-link problem and the related distancehop trade-off, whereby energy efficient geographic forwarding must strike a balance between shorter, high-quality links, and longer lossy links. The study is done for scenarios with and without automatic repeat request (ARQ).

Based on an analytical link loss model, we study the distance-hop trade-off via mathematical analysis and extensive simulations of a wide array of blacklisting/link-selection strategies; we also validate some strategies using a set of real experiments on motes. Our analysis, simulations and experiments all show that the product of the packet reception rate $(\mathrm{PRR})$ and the distance traversed towards destination is the optimal forwarding metric for the ARQ case, and is a good metric even without ARQ. Nodes using this metric often take advantage of neighbors in the transitional region (high-variance links). Our results also show that receptionbased forwarding strategies are more efficient than purely distance-based strategies; relative blacklisting schemes reduce disconnections and achieve higher delivery rates than absolute blacklisting schemes; and that ARQ schemes become more important in larger networks.
\end{abstract}

\section{Categories and Subject Descriptors}

C.2.1 [Network Architecture and Design]: Wireless communications; C.4 [Performance of Systems]: Perfor-

${ }^{*}$ This work has been supported in part by NSF under grants number 0347621 and 0134650, Intel, Pratt\&Whitney, and a gift grant from Ember Corporation.

Permission to make digital or hard copies of all or part of this work for personal or classroom use is granted without fee provided that copies are not made or distributed for profit or commercial advantage and that copies bear this notice and the full citation on the first page. To copy otherwise, to republish, to post on servers or to redistribute to lists, requires prior specific permission and/or a fee.

SenSys'04, November 3-5, 2004, Baltimore, Maryland, USA.

Copyright 2004 ACM 1-58113-879-2/04/0011 ...\$5.00. mance attributes; I.6 [Computing Methodologies]: Simulation and Modeling

\section{General Terms}

Performance, Design, Implementation

\section{Keywords}

Wireless Sensor Networks, Geographic Routing, Blacklisting

\section{INTRODUCTION}

Geographic routing is a key paradigm that is quite commonly adopted for information delivery in wireless ad-hoc and sensor networks where the location information of the nodes is available (either a-priori or through a self-configuring localization mechanism). Geographic routing protocols are efficient in wireless networks for several reasons. For one, nodes need to know only the location information of their direct neighbors in order to forward packets and hence the state stored is minimum. Further, such protocols conserve energy and bandwidth since discovery floods and state propagation are not required beyond a single hop.

The main component of geographic routing is usually a greedy forwarding mechanism whereby each node forwards a packet to the neighbor that is closest to the destination. This can be an efficient, low-overhead method of data delivery if it is reasonable to assume (i) sufficient network density, (ii) accurate localization and (iii) high link reliability independent of distance within the physical radio range.

However, while assuming highly dense sensor deployment and reasonably accurate localization may be acceptable in some classes of applications, it is now clear that assumption (iii) concerning highly reliable links is unlikely to be valid in any realistic deployment. Several recent experimental studies on wireless ad-hoc and sensor networks $[8,7,9,10]$ have shown that wireless links can be highly unreliable and that this must be explicitly taken into account when considering higher-layer protocols. Figure 1 (a) shows samples from a statistical link layer model developed in [16] — it shows the existence of a large "transitional region" where the link quality has high variance, including both good and highly unreliable links.

The existence of such unreliable links exposes a key weakness in greedy forwarding that we refer to as the weakest link problem. At each step in greedy forwarding, the neighbors that are closest to the destination (also likely to be farthest from the forwarding node) may have poor links with the current node. These "weak links" would result in a high rate of 


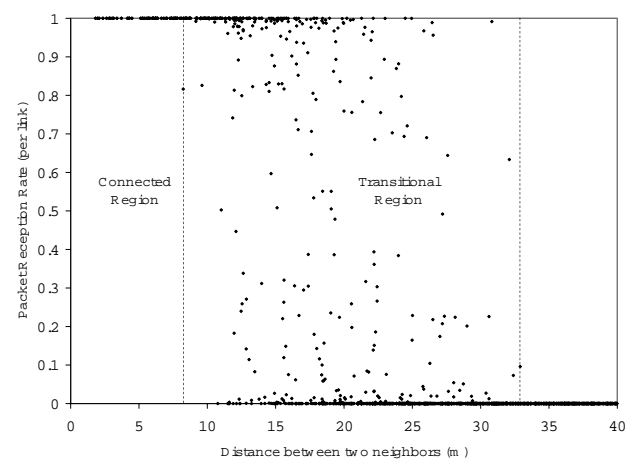

(a)

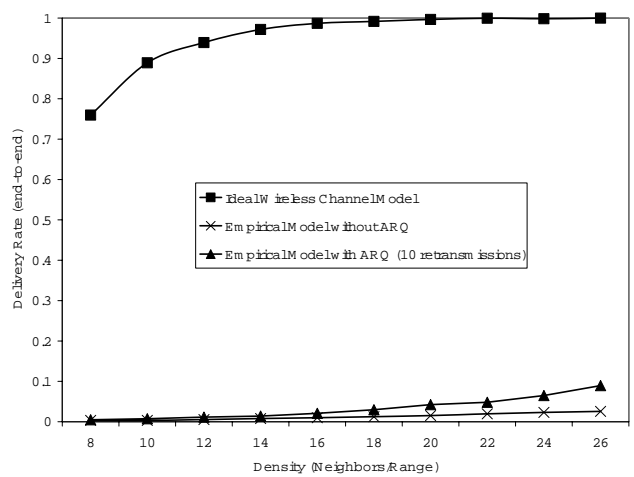

(b)

Figure 1: (a) Samples from a realistic analytical link loss model (b) An illustration of the discrepancy of performance of greedy geographic forwarding between an idealized perfect-reception model and the lossy reception model

packet drops, resulting in drastic reduction of delivery rate or increased energy wastage if retransmissions are employed. Figure 1 (b) illustrates the striking discrepancy between the performance of greedy forwarding on the realistic lossy network versus a network with an idealized reception model.

This observation brings to the fore the concept of neighbor classification based on link reliability. Some neighbors may be more favorable to choose than others, not only based on distance, but also based on loss characteristics. This suggests that a blacklisting/neighbor selection scheme may be needed to avoid 'weak links'. But, what is the most energyefficient forwarding strategy and how does such strategy draw the line between 'weak' and 'good' links?

We articulate the following energy trade-off between distance per hop and the overall hop count, which we simply refer to as the distance-hop energy trade-off for geographic forwarding. If the geographic forwarding scheme attempts to minimize the number of hops by maximizing the geographic distance covered at each hop (as in greedy forwarding), it is likely to incur significant energy expenditure due to retransmission on the unreliable long weak links. On the other hand, if the forwarding mechanism attempts to maximize per-hop reliability by forwarding only to close neigh- bors with good links, it may cover only a small geographic distance at each hop, which would also result in greater energy expenditure due to the need for more transmission hops for each packet to reach the destination. We will show in this paper that the optimal forwarding choice is generally to neighbors in the transitional region.

A related concern in designing a forwarding scheme is that it must be careful not to blacklist too many links, as this may result in route disconnections and lower delivery rates even in moderately dense networks.

In this work, our goal is to study the energy and reliability trade-offs pertaining to geographic forwarding in depth, both analytically and through extensive simulations, under a realistic packet loss model. For this reason, we utilize the statistical packet loss model derived in [16]. We emphasize, however, that the framework, fundamental results and conclusions of this paper are quite robust and not limited by the specific characteristics of this model. The main contributions of this work includes:

- Mathematical analysis of optimal forwarding choices to balance the distance-hop energy trade-off for both ARQ and No-ARQ scenarios.

- A framework to study and analyze greedy forwarding strategies in the context of geographic routing. The framework is applicable for other channel models as well, even though we apply it, in this study, to a specific set of channel parameters.

- Introduction of several new blacklisting and link-selection strategies suitable for geographic forwarding: based on distance, PRR and a combination of both. Then, we provide a systematic evaluation of those strategies via extensive simulations.

- The conclusion that PRR $\times$ distance is an optimal metric for making localized geographic forwarding decisions in lossy wireless networks with ARQ mechanisms, and is also a good metric for No-ARQ scenarios.

- Validation of this conclusion using a set of real experiments with motes to compare basic geographic forwarding approaches.

The rest of the paper is organized as follows. The related work is described in section 2 . In section 3 , we present the statistical link-loss model, scope and metrics of our work. Then, we provide a mathematical analysis of the optimum distance in the presence of unreliable links in section 4. A set of tunable geographic forwarding strategies suitable for lossy wireless networks is presented in section 5 . In section 6 , we report the results of a comprehensive simulation study that evaluates the performance of these strategies. The effectiveness of the PRR $\times$ distance metric is validated through real experiments with motes in section 7 . Finally, we discuss the implications of our results and future extensions of this work in section 8 .

\section{RELATED WORK}

Our study is informed by prior work on geographic forwarding and routing, as well as recent work on understanding realistic channel conditions and their impact on wireless network routing protocols. 
Early work in geographic routing considered only greedy forwarding [2] by using the locations of nodes to move the packet closer to the destination at each hop. Greedy forwarding fails when reaching a local maximum, a node that has no neighbors closer to the destination. A number of papers in the past few years have presented face/perimeter routing techniques to complement and enhance greedy forwarding $[1,3,5]$. We, however, set aside this issue for the present, and focus only on studying and suggesting ways to improve the greedy forwarding aspect of geographic routing. More details about geographic and position-based routing schemes can be found in the following surveys [12, 13].

On the other hand, much of the prior research done in wireless ad hoc and sensor networks, including geographic routing protocols, has been based on a set of simplifying idealized assumptions about the wireless channel characteristics, such as perfect coverage within a circular radio range. It is becoming clearer now to researchers and practitioners that wireless network protocols that perform well in simulations using these assumptions may actually fail in reality.

Several researchers have pointed out how simple radio models may lead to wrong results in wireless ad hoc and sensor networks. Ganesan et al. [7] present empirical results from flooding in a dense sensor network and study different effects at the link, MAC, and application layers. Some observations from their experiments are: the distribution of packet reception over distance is non-uniform; losses happen also at short distances from the transmitter; asymmetric links are quite common, especially at lower power settings. Kotz et al. [6] enumerate the set of common assumptions used in MANET research, and provide data demonstrating that these assumptions are not usually correct. The real connectivity graph can be much different from the ideal graph, and losses due to fading and obstacles are common at a wide range of distances and keep varying over time. The coverage area of radio is neither circular nor convex, and is often non-contiguous.

Zhao and Govindan [10] report measurements of packet delivery for a dense sensor network in different indoor and outdoor environments. Their measurements also point to a gray area within the communication range of a node, where there is large variability in packet reception over space and time. Similarly, the measurements obtained by the SCALE connectivity assessment tool [14] show that there is no clear correlation between packet delivery and distance in an area of more than $50 \%$ of the communication range (which corresponds to the transitional region we consider in our work). SCALE is also used in [15] to develop statistical models for characterizing links in sensor networks.

Several recent studies have shown the need to revisit routing protocol design in the light of realistic wireless channel models. In [8], De Couto et al. have measurements for DSDV and DSR, over a 29 node 802.11 b test-bed and show that the minimum hop-count metric has poor performance, since it is not taking the channel characteristics into account especially with the fact that minimizing the hop count maximizes the distance travelled by each hop, which is likely to increase the loss ratio. They present the expected transmission count metric that finds high throughput paths by incorporating the effects of link loss ratios, asymmetry, and interference. On the same line of work, Woo et al. [9] study the effect of link connectivity on distance-vector based routing in sensor networks. They too identify the existence of the three distinct reception regions: connected, transitional, and the disconnected regions. They evaluate link estimator, neighborhood table management, and reliable routing protocols techniques. A frequency-based neighbor management algorithm (somewhat related to the blacklisting techniques studied in our work) is used to retain a large fraction of the best neighbors in a small-size table. They show that cost-based routing using a minimum expected transmission metric shows good performance. The concept of neighbor management via blacklisting of weak links is also found in the most recent versions of the Directed Diffusion Filter Architecture and Network Routing API [17]. More recently in [11], empirical data is used to study the impact of radio irregularity in sensor networks. The results show that radio irregularity has more significant impact on routing protocols than on MAC protocols and that location-based protocols perform worse in the presence of radio irregularity than ondemand protocols.

However, we are not aware of any prior work that has studied the design of geographic routing schemes for lossy networks and has considered the related trade-offs in detail. Our work attempts to make a significant contribution in this direction. Although, the minimum expected transmission metric used in [8] and [9] is somewhat related to our $\mathrm{PRR} \times$ distance metric in trying to reduce the total number of transmissions from source to destination and thus minimize the energy consumed, the minimum expected transmission metric is a global path metric, while $\mathrm{PRR} \times$ distance is a local link metric suitable for scalable routing protocols such as geographic routing. Since in geographic routing, nodes need only to know the location information of their direct neighbors in order to forward packets, path metrics in general are not suitable for geographic routing protocols.

\section{MODEL, SCOPE AND METRICS}

Model: For both the analysis and simulations undertaken in this study, we required a realistic channel model for lossy sensor networks. Our work use the link layer model derived in $[16]$ :

$$
\operatorname{PRR}(d)=\left(1-\frac{1}{2} \exp ^{-\frac{\gamma(d)}{2} \frac{1}{0.64}}\right)^{\rho 8 f}
$$

Where $d$ is the transmitter-receiver distance, $\gamma$ is the signal to noise ratio (SNR), $\rho$ is the encoding ratio and $f$ is the frame length ${ }^{1}$. The model considers several environmental and radio parameters, such as the path-loss exponent $(\eta)$ and log-normal shadowing variance $(\sigma)$ of the environment, and the modulation and encoding schemes of the radio. This particular equation resembles a MICA2 mote, which uses non-coherent frequency shift keying as the modulation technique and Manchester as the encoding scheme $(\rho=2)$. The environmental parameters were set to $\eta=3.0$ and $\sigma=3.8$. Figure 1 (a) shows instances of the link layer model where the different regions can be observed. For this particular model the connected region goes from 0 to $8 \mathrm{~m}$, and the transitional region from 8 to $32 \mathrm{~m}$ approximately.

Scope: Radio activity (transmissions and receptions) has been identified as a major source of energy consumption in wireless sensor networks. This work provides a methodology to minimize the energy consumption during communication

\footnotetext{
${ }^{1}$ Please refer to [16] for a complete description of the model.
} 
events. Nevertheless, we should offer some caveats regarding the scope of our work. Our models do not consider other means of energy savings such as sleep/awake cycles, transmission power control, nor other sources of energy consumption such as processing or sensing. We also do not address MAC-layer behavior such as contention. We believe that this is a complementary and orthogonal problem to our work, since our model applies during the transmission/reception periods (at any snapshot of the topology), where the traffic and contention are relatively light; a very reasonable assumption for many classes of data-centric sensor networks.

Metrics: In order to evaluate the energy efficiency of different strategies, we use the following metrics:

- Delivery Rate $(r)$ : percentage of packets sent by the source which reached the sink.

- Total Number of Transmissions $(t)$ : total number of packets sent by the network, to attain the delivery rate described above.

- Energy Efficiency $\left(E_{e f f}\right)$ : number of packets delivered to the sink for each unit of energy spent by the network.

Our analysis is, as well, based on the following assumptions:

- Nodes know the location and the link's PRR of their neighbors.

- Nodes are randomly distributed.

$E_{\text {eff }}$ can be derived from the delivery rate $r$ and the total number of transmissions $t$. Let $p_{s r c}$ be the number of packets sent by the source, $e_{t x}$ and $e_{r x}$ the amount of energy required by a node to transmit and receive a packet, and $e_{r e}$ the energy used to read only the header of the packet (for early rejection). Given that we are assuming a random distributed topology, the expected number of neighbors can be considered as a constant $n$. Therefore, the total amount of energy consumed by the network for each transmitted packet is given by:

$$
e_{t o t a l}=e_{t x}+e_{r x}+(n-1) e_{r e}
$$

Subsequently, $E_{\text {eff }}$ is given by:

$$
E_{e f f}=\frac{p_{s r c} r}{k t}
$$

Where $k$ is a constant which includes $e_{\text {total }}$ and a conversion factor for energy units.

\section{ANALYTICAL MODEL}

Given a realistic link layer packet reception rate model, like the one described in section 3, our goal is to explore the distance-hop trade-off, in order to maximize the energy efficiency of the network during communication events. More precisely, we look into the following questions: at each step along the path, how far should a packet be forwarded? is there an optimal forwarding distance? The analytical models derived in this section provide an answer for these questions by obtaining the probability mass function $(\mathrm{pmf})$ of the optimal distance for architectures with and without automatic repeat request (ARQ).

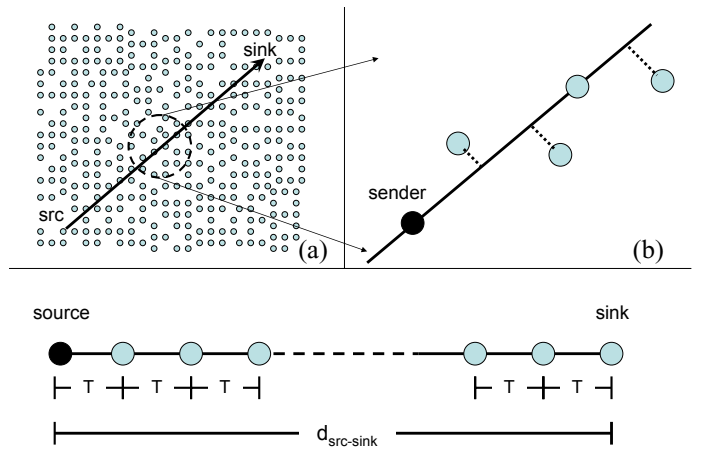

(c)

Figure 2: Derivation of the chain topology used in the mathematical analysis.

\subsection{Probabilistic Model for Distribution of Optimal Forwarding Distance}

This sub-section describes the notation and set-up used in the analytical models for systems with and without ARQ. Figure 2 (a) depicts a sensor network where the arrow shows the direction from source to sink. Figure 2 (b) is a zoom-in of some intermediate part of the path. Even though some nodes may not be aligned, the projection of their positions onto the arrow suggest how close the packet gets to the sink in case one of those nodes is chosen. Hence, the sourcedestination path can be described as a chain topology. Figure 2 (c) shows the chain topology used in our mathematical model. In this chain, nodes are placed every $\tau$ meters $^{2}$, the distance between source and sink is $d_{s r c-s i n k}$ and we want to obtain the optimal distance $d_{\text {opt }}$ to forward the packet.

For the remainder of this section, the analysis is focused in this chain topology and the following notation is used:

- $\eta$ : set of nodes that belong to the path between source and sink (including the source, but not the sink).

- $\gamma$ : set of links used in the path.

- $\psi$ : set of neighbors' distance ${ }^{3}$.

Our goal is to maximize $E_{e f f}$, for this purpose, let $X_{d}=$ $E_{e f f}(d)$ be a continuous random variable, which indicates the energy efficiency obtained if a distance $d$ is traversed at each hop. Hence, the goal can be re-stated as to find the distance that maximizes $X_{d}$. Let $q_{d}$ be the probability that a node at distance $d$ has the highest value of $X_{d}$ (i.e. $\left.X_{d}>X_{j}, \forall j \epsilon \psi, j \neq d\right)$. Thus, $q_{d}$ is the probability that the optimum forwarding distance is $\mathrm{d}$ and is given by:

$$
q_{d}=\int P\left(\left(x<X_{d}<x+d x\right) \wedge\left(X_{j}<x, \forall j \epsilon \psi, j \neq d\right)\right) d x
$$

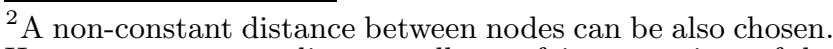
However, a constant distant $\tau$ allows a fair comparison of the different regions (connected, transitional, disconnected).

${ }^{3}$ In this section, neighbors are considered as nodes within $40 \mathrm{~m}$ of a sender.
} 


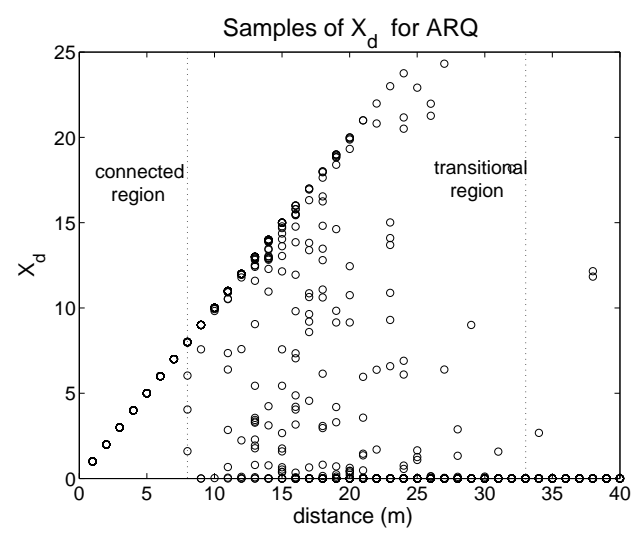

Figure 3: Energy efficiency metric for the ARQ case. The transitional region often has links with good performance as per this metric.

Since the PRR of a link $j, \forall j \epsilon \psi$, is considered independent ${ }^{4}$ :

$$
q_{d}=\int P\left(x<X_{d}<x+d x\right) P\left(X_{j}<x, \forall j \epsilon \psi, j \neq d\right) d x
$$

Finally, we get:

$$
q_{d}=\int f_{X_{d}}(x) \prod_{\forall j \in \psi, j \neq d} F_{X_{j}}(x) d x
$$

Where $f_{X_{d}}(x)$ and $F_{X_{j}}(x)$ are the $p d f$ and $c d f$ of the metric $X_{d}$, which represents the energy efficiency of a given forwarding strategy. In the next subsection, we evaluate equation (6) for the ARQ and No-ARQ cases.

\subsection{Analysis for ARQ case}

We assume no a-priori constraint on the maximum number of retransmissions (i.e. $\infty$ retransmissions can be performed), therefore, $r$ is equal to 1 . Consequently, according to equation (3), $E_{\text {eff } f}$ is given by:

$$
E_{e f f_{A R Q}}=\frac{p_{s r c}}{k t}
$$

At each hop the expected number of transmissions is $\frac{p_{s r c}}{P R R(d)}$, if distance $d$ is chosen. Therefore, the total number of transmission $t$ is given by $^{5}$ :

$$
t=p_{\text {src }} \frac{1}{\operatorname{PRR}(d)} \frac{d_{\text {src-sink }}}{d}
$$

Substituting $t$ in equation (7) we get:

$$
E_{e f f_{A R Q}}=\frac{P R R(d) d}{k d_{s r c-s i n k}}
$$

Thus, in order to maximize $E_{\text {eff }} f_{A R Q}$, we need to maximize the $P R R \times$ distance product. Hence, we define the metric $X_{d}$ as $P R R(d) \times d$. Figure 3 shows analytical samples obtained for $X_{d}$. For each distance, the PRR obtained was multiplied by its distance, it can be observed that nodes in the transitional region usually have the highest values of $X_{d}$.

\footnotetext{
${ }^{4}$ The received signal in a wireless channel is the sum of many contributions, coming from different locations, with random phases [18]. Therefore, we consider the PRR independent for different transmitter-receiver distances.

$5 \frac{d_{s r c-s i n k}}{d}$ in equation (8) represents the number of hops.
}

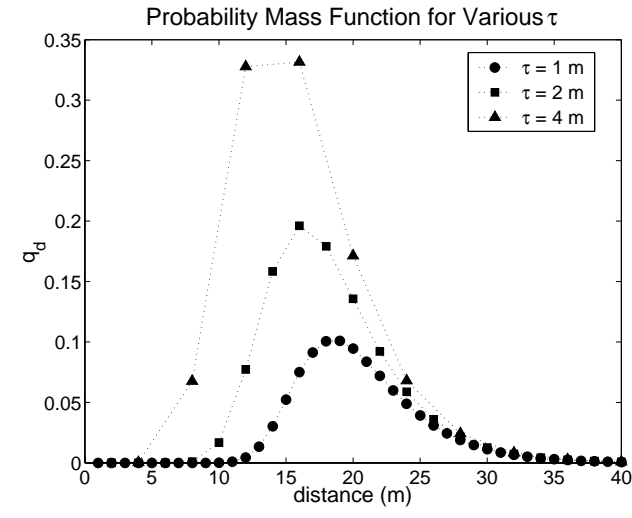

Figure 4: Probability mass function of the optimal forwarding distance in the ARQ case for different values of $\tau$.

The next step is to obtain the $c d f$ and $p d f$ for $X_{d}$ with the aim of evaluating the integral given in (6). Since the $\mathrm{PRR} \times$ distance product does not have a close-form expression, equation 6 was evaluated through numerical computation. After evaluating the integral for various values of $\tau$ (1, 2 and 4 meters), figure 4 shows the $p m f$ of the optimal ${ }^{6}$ distance. For small values of $\tau$ (1 and 2 ), which may correspond to high dense networks, nodes in the transitional region appear as the optimal ones, moreover, nodes in the connected region have negligible probabilities of being chosen. As $\tau$ increases $(4 \mathrm{~m})$, which may correspond to less dense networks, one node in the connected region $(8 \mathrm{~m})$ have a non-negligible probability of being optimal.

The analytical model derived provides the optimal distance. Nevertheless, there is an important question that must be solved to accurately evaluate the distance-hop tradeoff: how much are we gaining from choosing the best candidate? In other words, if we sort the nodes in the routing table according to the $\mathrm{PRR} \times$ distance metric (the highest the metric the better the candidate), how much energy are we saving by choosing the best candidate according to this metric instead of using other metrics, for example, the node with the highest PRR?.

Monte-Carlo simulations were performed to quantify the amount of energy savings. A chain topology was simulated with $d_{s r c-s i n k}=1000 \mathrm{~m}$, where a packet was sent from source to sink using four different forwarding policies.

- PRR $\times$ DIST: the packet is forwarded through neighbors that have the highest PRR $\times$ distance metric.

- BR: the packet is forwarded through the neighbor that has the highest PRR. If several nodes have the same PRR, the node closest to the sink is chosen.

- CONN: the packet is forwarded through the node in the connected region that is closest to the sink.

- GR (1\%): greedy forwarding through nodes that have PRR above $1 \%$.

\footnotetext{
${ }^{6}$ optimal refers to the local distance that statistically has the highest probability of providing the highest energy efficiency.
} 


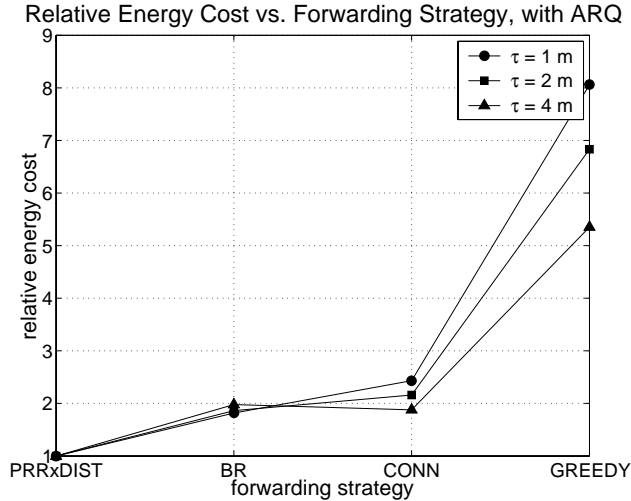

Figure 5: Relative energy efficiency of several forwarding strategies with respect to $P R R \times$ distance for the ARQ case.

The $E_{\text {eff }}$ is measured for the different strategies and compared relatively to the $E_{\text {eff }}$ of the PRR $\times$ DIST strategy. Therefore, each of the curves in Figure 5 shows how well the non-optimal forwarding schemes do relative to $\mathrm{PRR} \times \mathrm{DIST}$, and not absolute values of energy costs.

One hundred runs were performed for each value of $\tau$. Figure 5 shows that the curves for the different values of $\tau$ are similar, performing slightly better as $\tau$ increases. If the packet is forwarded through the best reception neighbor (BR), the network can consume as much as $100 \%$ more than the optimal (minimum) amount of energy. The figure also shows that the CONN strategy -which blacklists all nodes beyond the connected region- leads to energy inefficiencies of $100 \%$ or above. Finally, network protocols using greedy forwarding, with a low blacklisting threshold ${ }^{7}$, would consume energy above $500 \%$ of the optimal strategy. Due to space constraints a plot showing the probability distribution of the nodes used as forwarders is not presented, however, the nodes chosen in the PRR $\times$ DIST scheme show a similar distribution to the curves in Figure 4.

Finally, it is worth mentioning that correlations and variations in time of the PRR of a given link, may influence successive retransmissions and they form part of our future work. However, some of our empirical tests show that good and bad channel periods of a static link follow an exponential distribution (i.e. memoryless, small correlation) implying that the PRR over a relatively small period of time is a good estimator of the channel's condition.

\subsection{Analysis for the No-ARQ case}

This analysis is similar to the one in the previous subsection, with an important difference: in systems without $A R Q$, the distance between the source and the sink influences the election of the optimal forwarding distance.

In systems that uses ARQ, at each step a node transmits the same amount of data as the source, this characteristic allows us to do the analysis independently of $d_{\text {src-sink }}$. On the other hand, in systems without ARQ the amount of data decreases at each hop, hence the longer the $d_{\text {src-sink }}$ the higher the PRR of the chosen links should be. The analysis

\footnotetext{
${ }^{7}$ The low blacklisting threshold is to avoid choosing naively links with PRR less than $1 \%$.
}
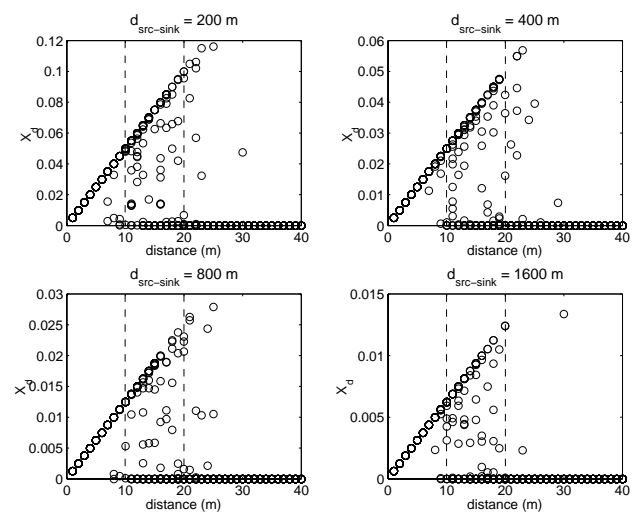

Figure 6: Energy efficiency metric for the No-ARQ case.

in this section explains this behavior.

In systems without ARQ, the delivery ratio $r$ is given by:

$$
r=\prod_{i \epsilon \gamma} P R R_{i}
$$

The number of packet transmissions required at each node $j$ that belongs to $\eta$ is given by:

$$
t_{j}=p_{\text {src }} \prod_{i=0}^{j-1} P R R_{i}
$$

Where $P R R_{0}=1$, to accommodate for the number of transmissions required at the source (equal to $p_{s r c}$ ). The total number of transmissions $t$ is the sum of $t_{j}, \forall j \in \eta$. Therefore $t$ is given by:

$$
t=p_{\text {src }} \sum_{j \in \gamma} \prod_{i=0}^{j-1} P R R_{i}
$$

The number of hops $h$ in the chain topology, defined for the analysis, is given by:

$$
h=\frac{d_{s r c-s i n k}}{d_{o p t}}
$$

Given that at each hop the packet traverses a distance $d$ with $P R R(d)$, equations (10) and (12) can be simplified to:

$$
\begin{gathered}
r=\operatorname{PRR}(d)^{h} \\
t=p_{\text {src }} \sum_{j=0}^{h-1} \operatorname{PRR}(d)^{j}
\end{gathered}
$$

For $P R R s<1$, the sum in the previous equation converges to:

$$
t=p_{s r c} \frac{P R R(d)^{h}-1}{P R R(d)-1}
$$

For $P R R s=1, t$ is given by:

$$
t=p_{\text {src }} h
$$

Finally, $E_{\text {eff }}$ is described by:

$$
E_{\text {eff } f_{w o A R Q}}= \begin{cases}\frac{P R R(d)^{h}}{\frac{k P R R(d)^{h}-1}{P R R(d)-1}} & , P R R<1 \\ \frac{1}{p_{s r c} h} & , P R R=1\end{cases}
$$




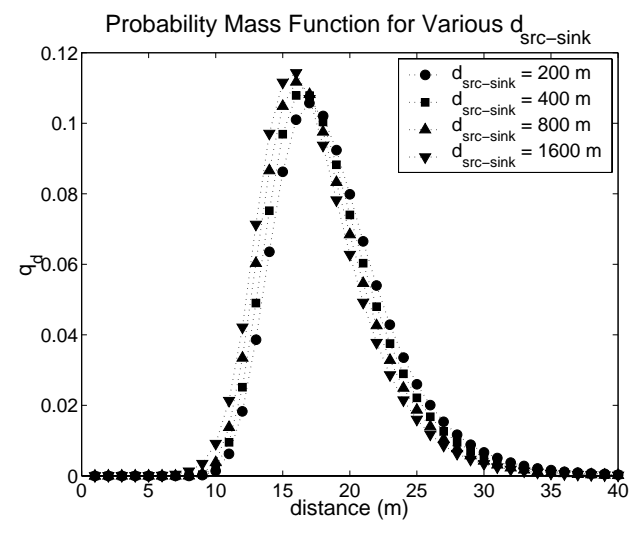

Figure 7: The probability mass function of the optimal forwarding distance for the No-ARQ case.

And $X_{d}$ is denoted by:

$$
X_{d}= \begin{cases}\frac{P R R(d)^{h} *(P R R(d)-1)}{P R R(d)^{h}-1} & , P R R<1 \\ \frac{1}{p_{s r c} h} & , P R R=1\end{cases}
$$

Similarly to the ARQ case, the $p d f$ and $c d f$ of $X_{d}$ do not have a simple tractable form. Therefore, numerical computation is used to approximate the integral given in (6).

The simulations for the No-ARQ case (figures 6 to 8 ) were done for only one value of $\tau(1 \mathrm{~m})$, on the contrary to the ARQ case where three values of $\tau$ were tested. The reason is that the interesting behavior in the No-ARQ case appears when $d_{\text {src-sink }}$ is modified, hence four scenarios were simulated; for $d_{\text {src-sink }}$ equals to $200,400,800$ and $1600 \mathrm{~m}$.

Figure 6 is the counterpart of Figure 3 for the $X_{d}$ metric, notice that the ARQ case required only one plot because its energy efficiency metric is independent of $d_{\text {src-sink }}$. We can observe two trends as $d_{s r c-s i n k}$ increases. First, the values of $X_{d}$ move slighty towards left (beginning of transitional region), indicating that higher PRRs are preferred for greater values of $d_{s r c-s i n k}$. Second, and rather obvious, the values of $X_{d}$ decrease; which is correct since, $X_{d}$ is directly proportional to $r$ and inversely proportional to $t$, and as $d_{s r c-s i n k}$ increases, $r$ decreases and $t$ increases.

Figure 7 shows the pmf of the optimal distance. As $d_{\text {src-sink }}$ increases the distribution starts moving slightly towards the beginning of the transitional region. The intuitive explanation is that for greater distances the delivery rate decreases to small values unless good links are chosen, and small delivery rates may imply small $E_{\text {eff }}$ 's.

Figure 8 compares the relative energy efficiency of three forwarding schemes $(\mathrm{PRR} \times \mathrm{DIST}, \mathrm{BR}$ and $\mathrm{CONN})$. BR and CONN have a similar performance for the different values of $d_{s r c-s i n k}$, showing an energy consumption of 70 to $80 \%$ more than the PRR $\times$ DIST strategy. The Greedy strategy was not included in the plot, since it performs several orders of magnitude worse, this low performance for the No-ARQ case is due to the dramatic decrease of the delivery rate if some of the links have low PRRs $(\sim 1 \%)$.

The analysis provided us with useful insights. First, the optimal forwarding decision based on the $\mathrm{PRR} \times$ distance metric in the ARQ case and equation (18) in the No-ARQ case, strikes a balance between the two extremes of forwarding to the farthest (likely worst reception) neighbor and the

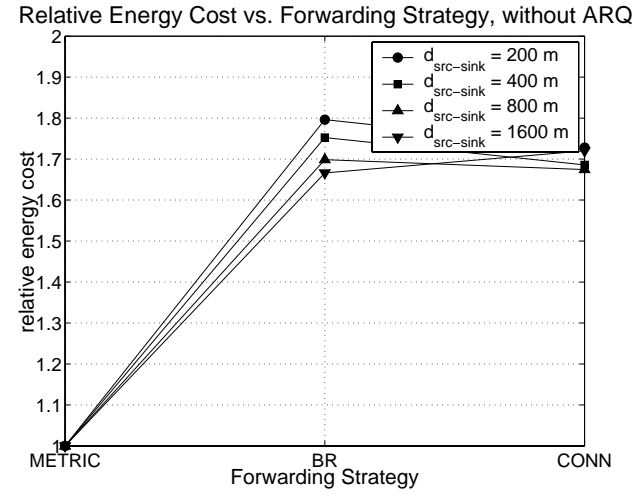

Figure 8: Relative energy efficiency of different forwarding strategies with respect to the optimal metric (equation 19) for the No-ARQ case.

nearest (likely best reception) neighbor. And second, the best candidate neighbor for forwarding often lies in the transitional region. However, there are some limitations in the analysis, it assumes a chain topology and that there are no network disconnections (which can be a problem in lowdensity scenarios when blacklisting is employed). In the next two sections we present a set of practical geographic strategies, and evaluate them in more realistic contexts through extensive simulations.

\section{GEOGRAPHIC FORWARDING STRATE- GIES FOR LOSSY NETWORKS}

In this section, we describe the design of novel forwarding strategies to improve the performance of geographic routing in lossy networks. In the next section, we will examine these strategies through a comprehensive simulation study. We classify our policies into two categories: distance-based and reception-based policies. In distance-based policies nodes need to know only the distance to their neighbors, while in reception-based policies, in addition to the distance, nodes need to know also the packet reception rates of their neighbors. As we will show in our study, using the reception rate information allows nodes to make better choices in forwarding the packet than choosing neighbors based only on distance, but it requires the extra functionality for obtaining the reception rate. In the coming discussion we will assume that the reception rate is available. Individual nodes can estimate the reception rate by monitoring the channel and observing packet success and loss events (refer to [8,9] for more details). Beacons sent by nodes for neighbor detection and communicating location information could also be used for assessing the reception rate by counting the number of beacons received from a neighbor within a period of time and sending this value to that neighbor.

All of our strategies use greedy-like forwarding, in that the next hop is chosen from the neighbors closer to the destination (or sink) than the forwarding node. As we mentioned, the original greedy forwarding approach, used in the currently proposed geographic protocols, forwards to the neighbor closest to the destination, which is likely to be at a large distance from the forwarding node and hence is likely to have a weak link to that node. In order to avoid 
nodes with weak links, we present blacklisting approaches that first blacklist a set of neighbors based on a certain criteria, and then forwards to the node closest to the destination among the remaining neighbors. We first present distancebased blacklisting based on the idea that neighbors above a certain distance are likely to have weak links. Distancebased blacklisting does not take the reception rate of neighbors into account which limits its performance, therefore we design reception-based blacklisting approaches in order to classify nodes based on the quality of their links. Absolute reception-based blacklisting avoids all neighbors below a certain reception rate, which guarantees links with a certain quality, but it risks greedy routing failures when a node has no neighbors closer to the destination above this reception rate. This risk of disconnection brings up the relative blacklisting strategy, which orders nodes closer to the destination based on a certain criteria and keeps the best of these nodes independent of their specific values, then it forwards to the node closest to the destination among those. In addition to the blacklisting approaches, we present two other forwarding schemes which rank nodes closer to the destination according to a specific metric. In the first approach, the metric is to pick the node with the highest reception rate (highest $\mathrm{PRR}$ ), and in the second approach, we use the metric promoted by our analysis and pick the node with the highest $\mathrm{PRR} \times$ distance. The following subsections explain our policies in more detail.

\subsection{Distance-based Forwarding}

Original Greedy: Original greedy is similar to the current forwarding policy used in common geographic routing protocols. Each node forwards to the neighbor closest to the destination from the set of neighbors that are closer to the destination. Since most of the previous studies were simulation-based using the ideal channel model, the neighbors were all the nodes within a certain radio range. In reality, a node will detect its neighbors when it hears their messages, therefore a minimum reception rate is required between two nodes in order for them to be considered neighbors. In the simulations, we set the minimum reception rate to $1 \%$. Original greedy is a special case of the coming blacklisting policies, when no nodes are blacklisted.

Distance-based Blacklisting: In this case, each node blacklists neighbors that are above a certain distance from itself. In the simulations we set the blacklisting threshold as a fraction of a nominal radio range. For example if the radio range is considered to be $40 \mathrm{~m}$ and the blacklisting threshold is $20 \%$, then the farthest $20 \%$ of the radio range $(8$ $\mathrm{m}$ ) is blacklisted and the packet is forwarded to the neighbor closest to the destination from those neighbors at a distance less than $32 \mathrm{~m}$ from the current forwarding node. Notice that actually no exact radio range value is required. The loss model distribution (Figure 1 (a)) could be used to determine the distance threshold.

\subsection{Reception-based Forwarding}

Absolute Reception-based Blacklisting: In absolute reception-based blacklisting, each node blacklists neighbors that have a reception rate below a certain threshold. For example, if the blacklisting threshold is $20 \%$, then only neighbors closer to the destination with a reception rate above $20 \%$ are considered for forwarding the packet and the closest neighbor to the destination among those is chosen.
Relative Reception-based Blacklisting: In relative reception-based blacklisting, when a node receives a packet, the node blacklists, from its neighbors that are closer to the destination, a percentage of nodes that have the lowest reception rate. For example, if the blacklisting threshold is $20 \%$, after a node receives a packet, it considers only the $80 \%$ highest reception rate neighbors of its neighbors that are closer to the destination, and it forwards the packet to the neighbor closest to the destination among those. Note that relative blacklisting is also different from the previous blacklisting methods in that the neighbors blacklisted are different for every destination, since blacklisting of a node depends on the node rank within a set of neighbors and there is no specific threshold value. Relative Blacklisting has the advantage of avoiding the disconnections that can happen in previous methods where all neighbors could be blacklisted, but it also risks having bad neighbors that may be wasteful to consider. By lowering the threshold, more neighbors are considered from which a neighbor with high distance could be selected. Higher thresholds will limit the choice only to higher PRR neighbors. Relative Reception-based thresholds provide a range between two extremes: Original Greedy which selects the highest distance independent of the PRR, and Best Reception which selects the highest PRR independent of distance.

Best Reception Neighbor: Each node forwards to the neighbor that has the highest PRR from the neighbors that are closer to the destination. This is a special case of relative reception-based blacklisting, where only a single node is considered and hence the distance is not affecting the choice.

Best PRR $\times$ distance: This is the metric shown in our analysis. For each neighbor that is closer to the destination, the product of the reception rate and the distance improvement achieved by forwarding to this neighbor is computed, and the neighbor with the highest value is chosen. In the analysis, we were using a chain topology, so the distance improvement was simply the distance between the forwarding node and the neighbor. In the simulations we are using random general topologies, so each node forwarding a packet computes the distance improvement to a neighbor as $1-\frac{d(n b r, d s t)}{d(n o d e, d s t)}$, where $d(n b r, d s t)$ is the distance between that neighbor and the destination, and $d$ (node,dst) is the distance between the node itself and the destination (this reflects how closer the packet gets to the destination by forwarding to that neighbor).

\section{SIMULATION RESULTS}

In this section, we perform extensive simulations to study the characteristics of different forwarding strategies in random topologies under different densities and network sizes. We use the packet reception model presented in section 3 . In this paper, we are mainly interested in evaluating the effects of physical-layer packet losses on geographic routing independent of the MAC layer used and without concerning ourselves with extraneous factors such as MAC collisions.

We simulate random static networks of sizes ranging from 100 to 1000 nodes having the same radio characteristics. We represent the density as the average number of nodes per a nominal radio range and vary it over a wide scale: 25 , 50, 100, 200 nodes/range. Notice that our link loss model does not have a strict value for the radio range similar to the values used for ideal channel models. Nonetheless, we 
define a nominal radio range in order to show the density as a function of that range, and set it to $40 \mathrm{~m}$ based on the distribution in Figure 1 (a). This density could be considered as the number of neighbors within the range of a node if an ideal channel model is used. But in this model, the nodes within the range of a node are not all neighbors (in terms of connectivity) of that node and this is why the density in terms of nodes/range is relatively high, while the actual neighbors are much less. For a node to be considered a neighbor it must have at least $1 \%$ reception rate.

In each simulation run, nodes are placed at random locations in the topology and 100 packet transmissions are issued from a random source to a random destination in the network. The results are computed as the average of 100 runs. By using random node distributions with different densities, we cover the effect of disconnections in a wide range of scenarios. At high densities, nodes are more uniformly distributed, and the greedy disconnections ${ }^{8}$ will be low (similar to our analysis and real experiments sections). While at lower densities, the distribution is more irregular and the space contains gaps, causing more greedy disconnections. The random topologies generated have a mix of distributions with some areas uniform and some areas containing gaps of different sizes. The tendency to higher uniformity or gaps depends on the density.

During packet transmission, the packet header contains the destination location and each node chooses the next hop based on the routing policy used. When a node forwards a packet to its neighbor, the probability that the packet is received is equal to the packet reception rate. The packet reception rate between two nodes is computed by applying their distance into the loss model equation. If the packet is dropped, the response depends on whether ARQ is used or not. If $\mathrm{ARQ}$ is not used, this packet is lost and not delivered to the destination. If ARQ is used, the packet is retransmitted again until it is received by that neighbor or a maximum retransmission count is reached. Our evaluations contain link layers without ARQ, with 10 retransmissions ARQ, and with infinite retransmissions ARQ. Since the minimum reception rate for a node considered as a neighbor is $1 \%$, infinite retransmissions are guaranteed to succeed.

The performance metrics studied are the delivery rate, the total number of transmissions, and the energy efficiency (bits/unit energy). The delivery rate (same as $r$ in the analysis) is the percentage of packets sent by the source and received by the destination. The total number of transmissions is the number of transmissions generated to deliver a packet between a source and a destination, and it contains all intermediate transmissions and retransmissions till the packet is delivered to the destination or is lost (this is the same as $t$ in the analysis). The bits/unit energy is the ratio of the delivery rate to the number of transmissions (same as $E_{e f f}$ in the analysis), and we use it to represent the amount of delivery contribution by each transmission, thus it represents the energy efficiency of the policy used (its inverse can be viewed as the amount of energy required to achieve a certain delivery rate).

We have done extensive simulations along various parameters for the different forwarding strategies. Due to space restrictions, we present here only some of the key results.

\footnotetext{
${ }^{8}$ We mean by greedy connectivity the existence of a path between the source and destination that can be detected by greedy forwarding.
}

In the coming sections, we will first show the results for the blacklisting strategies: distance-based, absolute receptionbased, and relative reception-based at an entire range of blacklisting thresholds and different densities. The goal is to identify the optimum thresholds and their characteristics. Then we will compare the different strategies by picking the optimum blacklisting threshold for each density and include also the original greedy, best reception policy, and the best $\mathrm{PRR} \times$ distance policy in the comparison. Then we will show the results at various distance ranges between source-destination pairs and compare the different policies at these ranges. Finally, we present some insights on the effects of ARQ and network size on the policies.

\subsection{Blacklisting Strategies}

In this subsection, we study distance-based, absolute receptionbased, and relative reception-based blacklisting strategies at different densities ${ }^{9}$. We vary the blacklisting threshold and show its effect on the delivery rate and energy efficiency. We use networks of 1000 nodes and set the number of ARQ retransmissions to 10 .

Figure 9 (a) and (b) show the delivery rate and bits/unit energy for distance-based blacklisting. The optimum blacklisting thresholds are within the transitional region which conforms with our analysis. The delivery rate is low at low thresholds, because of forwarding on low reception rate links that cannot deliver the packet even with 10 retransmissions. At high thresholds the delivery rate decreases again because of the increase in greedy disconnections that happen when all nodes closer to the destination are blacklisted. The blacklisting threshold has a trade-off between the quality of the link, the number of hops in the path between source and destination, and the greedy connectivity. Using low thresholds increases the possibility of forwarding on high-loss links causing the packet to be dropped. On the other hand, higher thresholds reduce the connectivity and increase the chances of greedy failures. Greedy disconnection is an important factor that was not captured in our analytical model that assumed very high density. As the density gets lower, the optimum threshold shifts to the left, since at lower densities the possibility of greedy disconnections is higher. The bits/unit energy also decreases at higher thresholds because of the wasted overhead of transmitting packets over multiple hops before being dropped due to greedy disconnections, in addition to the distance-hop energy trade-off. It is instructive to notice that at low densities, increasing the threshold does not cause much improvement over the original greedy, which indicates that forwarding based on distance only is very limited in improving the performance.

Figure 10 (a) and (b) show the delivery rate and bits/unit energy for absolute reception-based blacklisting. Forwarding based on reception rate provides in general higher delivery rates and bits/unit energy than forwarding based only on distance. A sharp increase in delivery rate happens at $10 \%$ threshold since 10 retransmissions on average are adequate to deliver the packet between two neighbors when the reception rate is above $10 \%$. This hints that, in general, the reception-based threshold should be set based on the number of retransmissions to make them at least higher than the average retransmissions required. At higher densities (100

\footnotetext{
${ }^{9}$ The density is shown as the number of nodes per nominal range. The actual number of neighbors could be much less and depends on the loss model.
} 


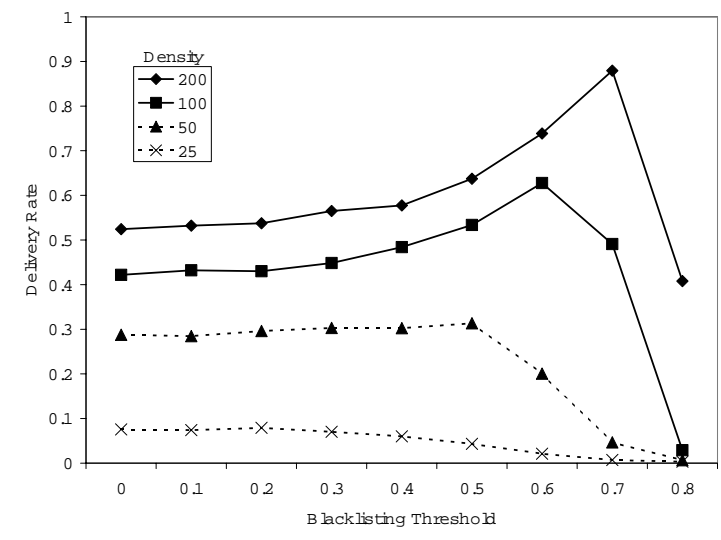

(a) Delivery Rate

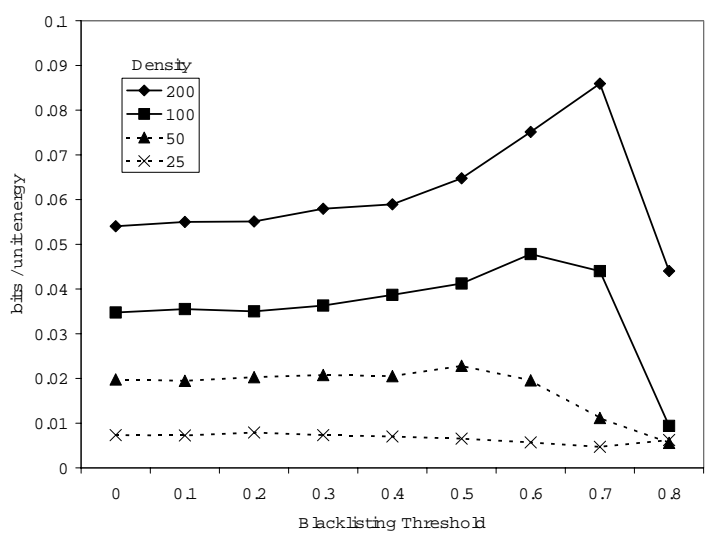

(b) Energy Efficiency

Figure 9: Performance of Distance-based Blacklisting Schemes for Geographic Forwarding

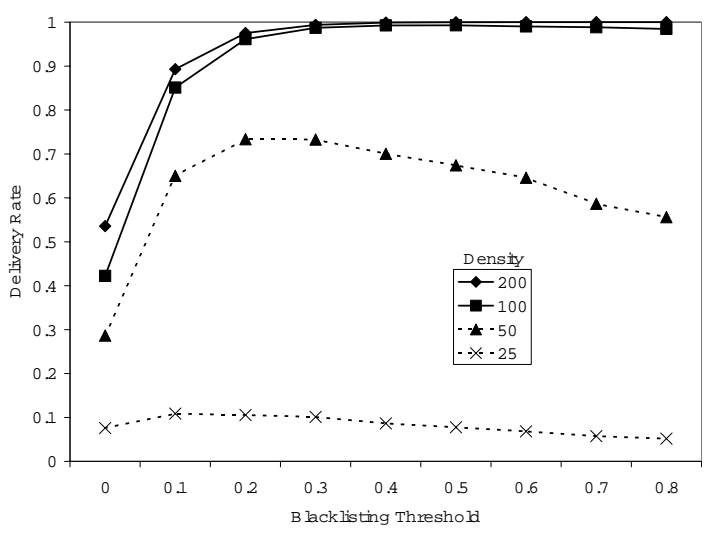

(a) Delivery Rate

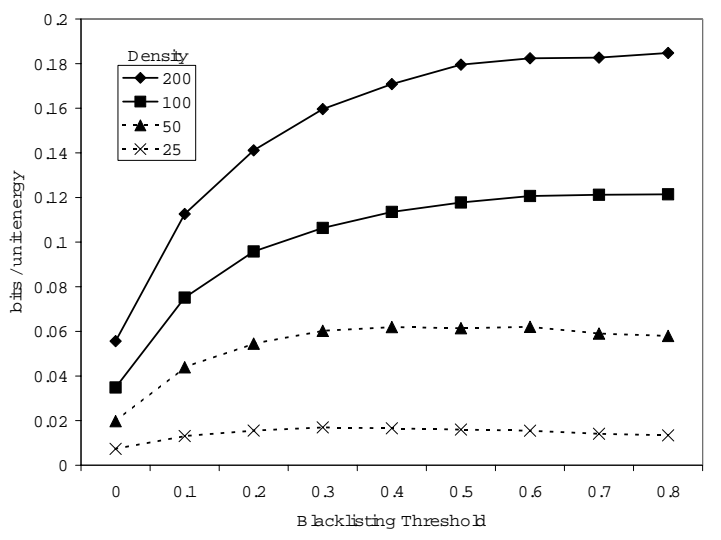

(b) Energy Efficiency

Figure 10: Performance of Absolute Reception-based Blacklisting Schemes for Geographic Forwarding

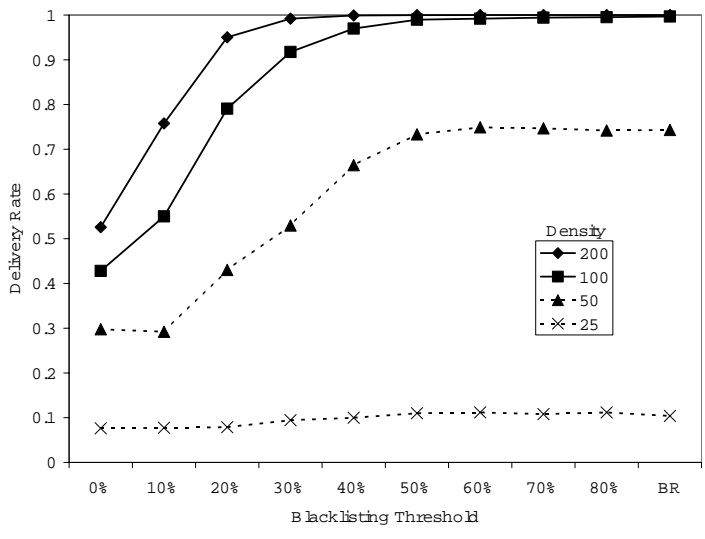

(a) Delivery Rate

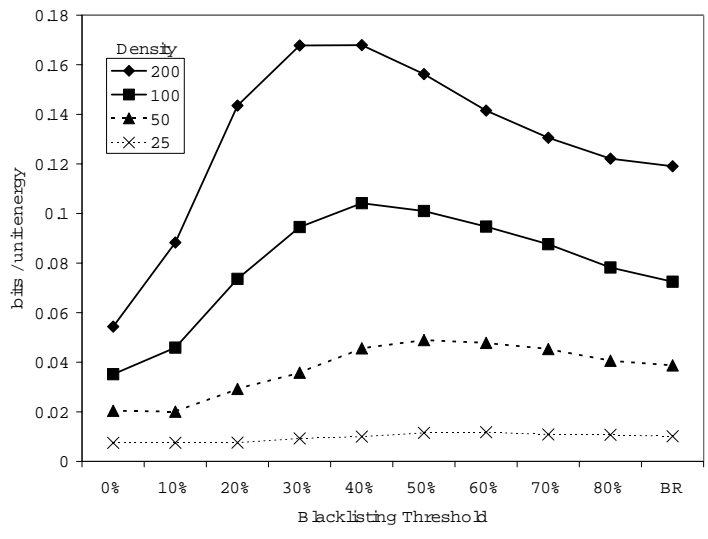

(b) Energy Efficiency

Figure 11: Performance of Relative Reception-based Blacklisting Schemes for Geographic Forwarding 
and 200), higher thresholds increase the delivery rate since better links are used and the possibility of disconnections is low. While at lower densities $(25,50)$, greedy disconnections cause the delivery rate to start dropping at low blacklisting thresholds. The bits/unit energy is also a balance between more retransmission overhead at low thresholds, and the disconnections and longer paths at high thresholds.

In Figure 11 (a) and (b) we show the delivery rate and bits/unit energy for relative reception-based blacklisting. Best Reception (BR) is also included as an extreme of relative blacklisting. The main merit of relative blacklisting is that it reduces disconnections by using the best available links independent of their quality or distance. We notice that at all densities, higher thresholds improve the delivery rate since better links are used with lower risk of increasing the greedy disconnections. Figure 11 (b), shows that increasing the blacklisting threshold improves the bits/unit energy, because better links are used which reduces the retransmission overhead. The increase in bits/unit energy is up to a certain value, and then the energy efficiency drops again due to the distance-hop energy trade-off. At high thresholds a small number of neighbors with high reception rates are likely to be chosen, but these neighbors are probably close to the forwarding node. By reducing the threshold, more nodes with high reception rates could be chosen with a larger variety of distances and a larger possibility to pick a neighbor much closer to the destination. This indicates also that just choosing the node with the best reception rate, may not be the most energy efficient approach. Relative blacklisting reduces greedy disconnections by choosing the best available nodes independent of their quality, but sometimes this causes bad links to be used which reduces the energy efficiency compared to absolute reception-based.

We should note that the threshold values of different blacklisting methods are not comparable, since they lead to different number of neighbors, link qualities, and neighbor distances. We note also that the optimum thresholds and in general the optimum strategies with regard to energy efficiency may not provide the optimum delivery rate and may not be satisfactory to provide the required connectivity.

\subsection{Comparison of Forwarding Strategies}

In this subsection, we compare the delivery rate and energy efficiency of our forwarding strategies at different densities and distance ranges. We also use networks of 1000 nodes and 10 retransmissions. For distance-based, absolute reception-based, and relative reception-based blacklisting, we use the optimum energy efficient threshold at each density, obtained from the previous subsection. In Figure 12 (a) and (b) we show the delivery rate and energy efficiency at different densities. The delivery rate is low at low densities, because of greedy failures. $\mathrm{PRR} \times$ distance has the highest delivery rate, followed by Best Reception, relative receptionbased, absolute reception-based, distance-based, and finally Original Greedy. The relative strategies $(\mathrm{PRR} \times$ distance, Best Reception, and relative reception-based) have the highest delivery rate, because they reduce greedy disconnections. Strategies based on reception rate are better than those based only on distance. PRR $\times$ distance and absolute reception-based blacklisting are the most energy efficient, followed by relative reception-based, Best Reception, distance-based, and finally Original Greedy. Higher densities improve both the delivery rate and energy efficiency.
In Figure 13 we show the results for different distance ranges. In the previous results we have shown the average performance in delivering packets between random sourcedestination pairs. Since, the performance may depend on the traffic pattern and the distances between the expected sources and destinations, we study here the effect of different patterns, by categorizing source-destination pairs based on their distance and examining the delivery rate and bits/unit energy at different distance ranges. We fix the density to an average of 50 nodes/range. We notice that the delivery rate and the energy efficiency decrease as the distance range increase, since more hops will be required and the probabilities of packet drops and greedy disconnections become higher. The order of the forwarding strategies remains the same as in the previous comparison.

The comparisons in this subsection show that $\mathrm{PRR} \times$ distance is a very effective strategy conforming with our analysis. It is mostly the highest for both delivery rate and energy efficiency. $\mathrm{PRR} \times$ distance is also much more robust and easier to implement, since no density-dependent absolute threshold parameter is required. Best Reception has a high delivery rate, but its energy efficiency is relatively lower due to the distance-hop energy trade-off. Conversely, absolute reception-based has relatively high energy efficiency, since it avoids wasting overhead on links with low reception rates, but its delivery rate is lower due to greedy disconnections.

\subsection{Effects of ARQ and Network Size}

We have shown simulation results for ARQ with 10 retransmissions, since we believe that ARQ with a limited number of retransmissions is normally the practical choice for implementation. In this subsection we compare ARQ with 10 retransmissions to transmission without ARQ and to ARQ with infinite retransmissions. We set the density to 50 nodes/range and vary the network size to observe the effect of ARQ on different network sizes. We show the results for Original Greedy and PRR $\times$ distance.

Figure 14 shows that the delivery rate of Original Greedy increases by using more retransmissions and since the lowest reception rate of a neighbor is $1 \%$, infinite retransmissions do not cause delivery failures due to losses, but delivery failures could still happen due to greedy disconnections. On the other hand, the energy efficiency of Original Greedy degrades with more retransmissions, due to the extra overhead of retransmitting on bad links. This shows that dealing with bad links by just using more retransmissions may improve the delivery rate, but at a cost of a very high energy and bandwidth wastage.

In Figure 15, the delivery rate of $\mathrm{PRR} \times$ distance improves, as expected, with more retransmissions, with a high improvement achieved from 0 to 10 retransmissions. By considering the reception rate in forwarding the packets and using better links, the number of retransmissions required for a certain delivery rate can be significantly reduced. The energy efficiency of 10 retransmissions ARQ is the highest, since it has a high delivery rate (only slightly lower than infinite ARQ) and its overhead is limited. A satisfactory number of retransmissions will depend on the loss rates and the delivery rate required. We notice that ARQ becomes more important as we increase the network size, which is also indicated by our analysis. The reason is that without ARQ, the probability of delivering a packet between a source and a destination over more hops decreases faster compared 


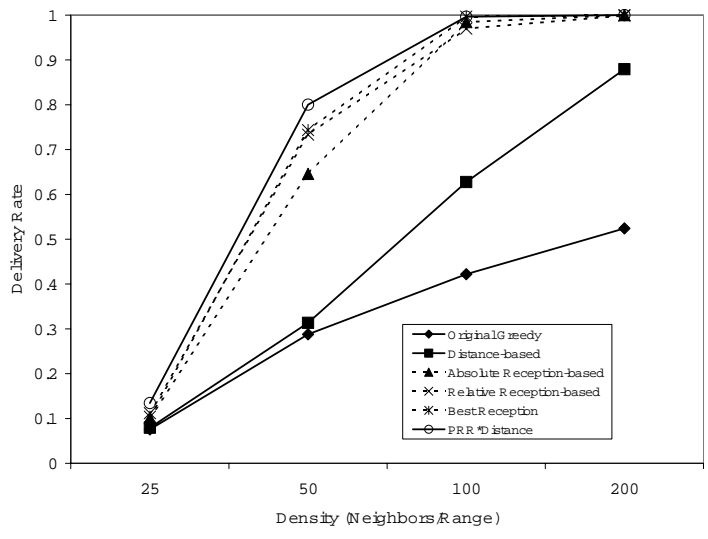

(a) DeliveryRate

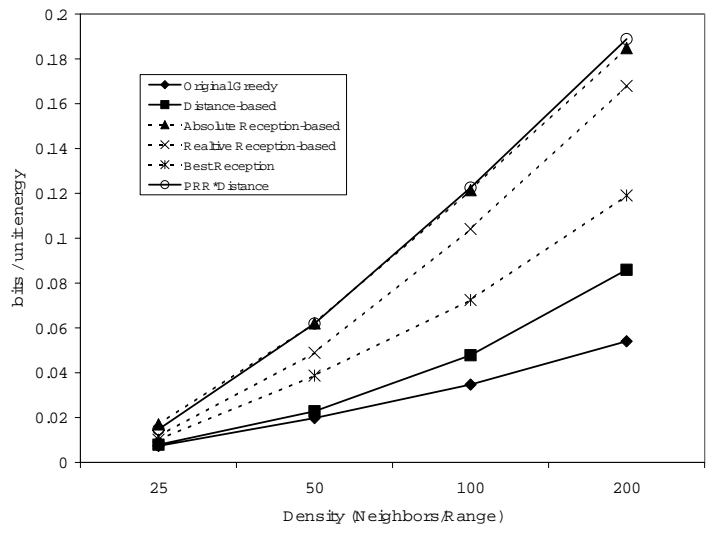

(b) Energy Efficiency

Figure 12: Performance of Geographic Forwarding Strategies at Different Densities

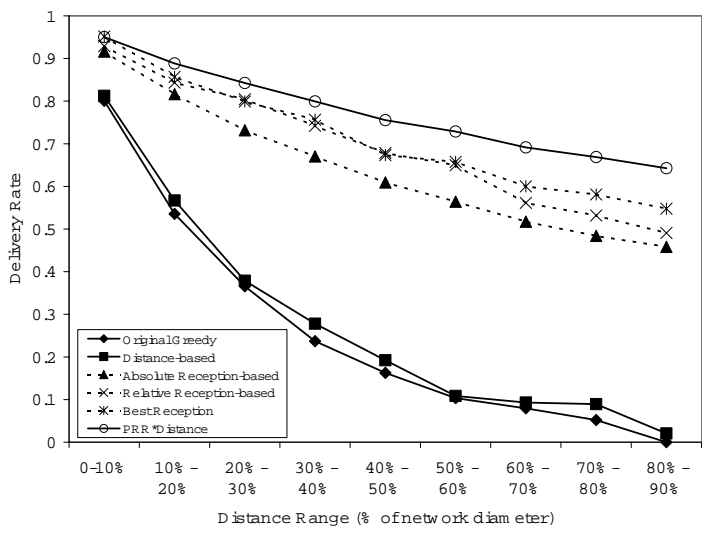

(a) DeliveryRate

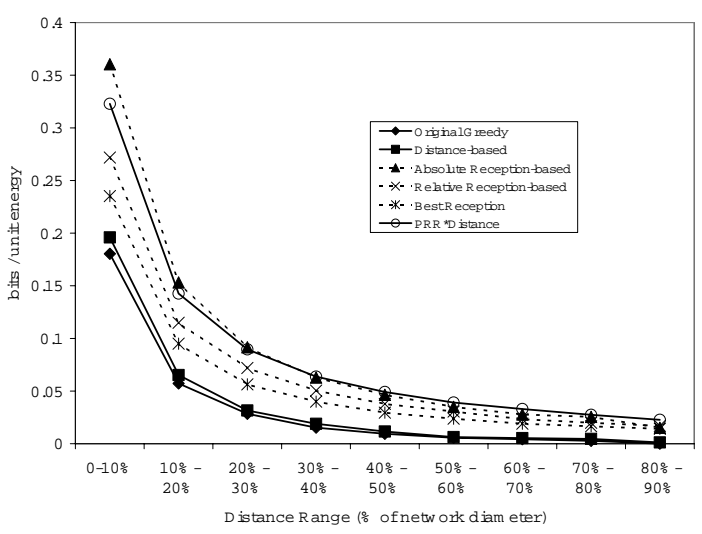

(b) Energy Efficiency

Figure 13: Performance of Geographic Forwarding Strategies at Different Source-Destination Distances. Each $10 \%$ distance range corresponds to about 1.5 times the nominal radio range $(40 \mathrm{~m})$

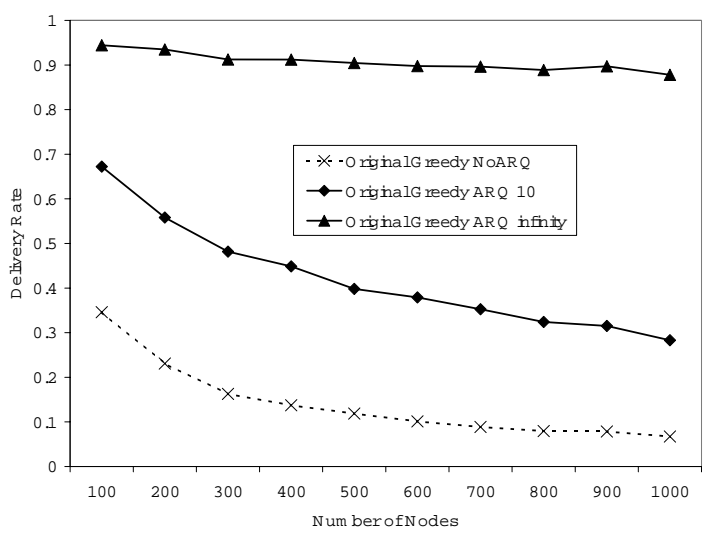

(a) DeliveryRate

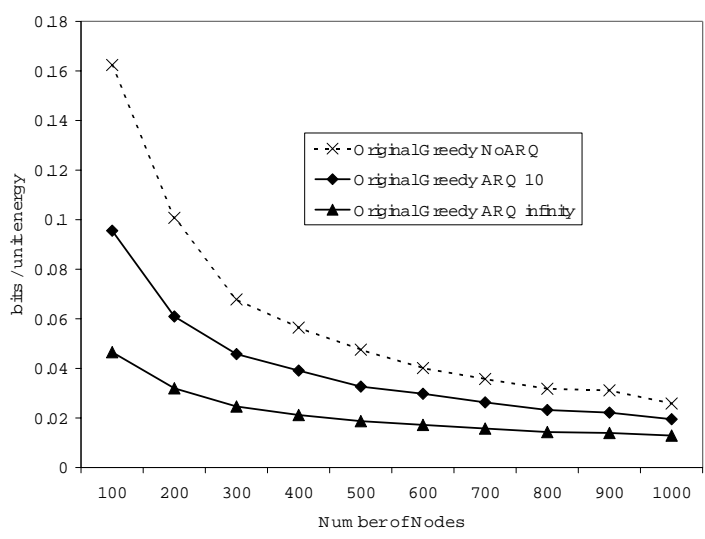

(b) Energy Efficiency

Figure 14: Performance of Original Greedy with and without ARQ at Different Network Sizes 


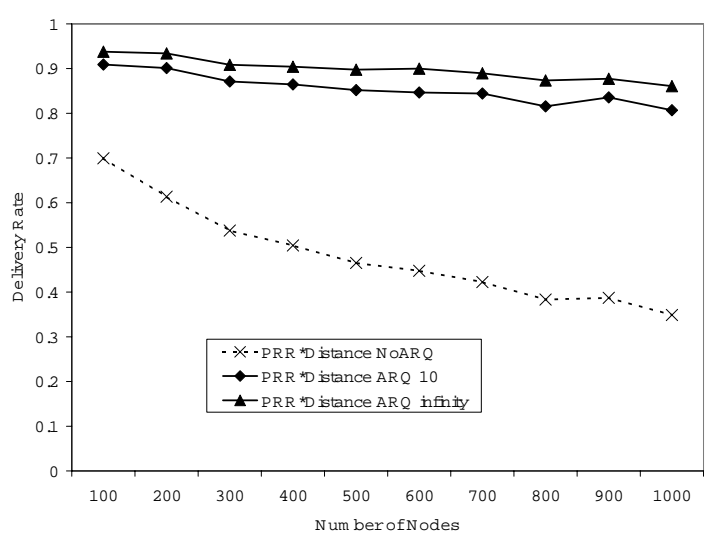

(a) DeliveryRate

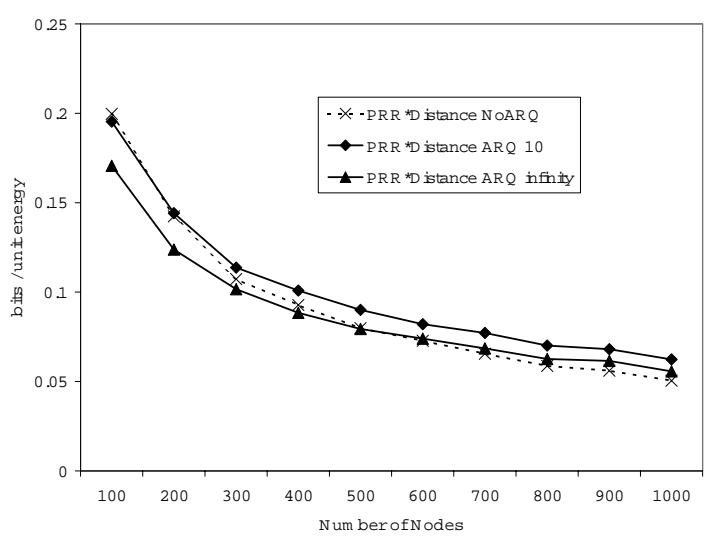

(b) Energy Efficiency

Figure 15: Performance of PRR $\times$ distance with and without ARQ at Different Network Sizes

to ARQ. In addition, there is extra wasted overhead due to delivering packets over more hops before being dropped.

\section{REAL EXPERIMENTS WITH MOTES}

In order to validate our methodology and conclusions, we undertook an experimental study on motes. Twenty-one (21) MICA2 motes were deployed in a chain topology spaced every $60 \mathrm{~cm}(\sim 2$ feet). The source (node 0$)$ and sink (node 20) were placed at opposite extremes of the chain. The power level was set to $-20 \mathrm{dBm}$ and the frame size was 50 bytes. Three different forwarding strategies were tested:

- GR: original greedy routing choose the neighbor closer to the sink whose $P R R>0$.

- BR: neighbor with highest PRR. In case two or more neighbors have the same PRR, the one closer to the sink is chosen.

- PRR $\times$ DIST: neighbors are classified according to the $\mathrm{PRR} \times$ distance metric.

First, the motes exchange test packets to measure the PRR of the links and populate their routing tables accordingly. Afterwards, the source sends 50 packets to the sink for each of the 3 different strategies (150 total). A maximum of 5 transmissions ( 1 transmission +4 retransmissions) are allowed at each hop, if the packet is not received after the fifth attempt, it is dropped.

Six different scenarios are studied: a football field, an indoor-building environment and four different outdoor-urban areas. The channel characteristics of some scenarios are significantly different, and hence, instead of providing a cumulative result, we present the results for each one of them.

Table 1 shows the delivery rate $(r)$, the number of transmission $(t)$, and the energy effciency $\left(E_{e f f}\right)$ for the different scenarios. BR have an $r$ of $100 \%$ in all scenarios, and PRR $\times$ DIST have $100 \%$ for all scenarios except scenario- 4 $(82 \%)$. Greedy performs poorly in most of them with zero or close to zero $r$ in most cases.

With regard to the number of transmissions, BR requires more transmissions than $\mathrm{PRR} \times \mathrm{DIST}$ in all scenarios except scenario 2 where BR performs better. Given that $r$ is similar for $\mathrm{BR}$ and $\mathrm{PRR} \times \mathrm{DIST}$, the difference in the energy efficiency is determined by the number of transmissions. BR consumes between 2 to $25 \%$ more energy than PRR $\times$ DIST, only in scenario- $2 \mathrm{PRR} \times \mathrm{DIST}$ performed $4 \%$ worse. On the other hand, in the two scenarios where Greedy has a non-zero $r$, it consumed $3 \%$ and $54 \%$ more energy than $\mathrm{PRR} \times \mathrm{DIST}$. It is interesting to observe that the energy "wasted" by greedy forwarding depends on where the first weak link is encountered. In some scenarios the first weak link is at the beginning of the chain and hence the energy wasted is not significant, however, in other scenarios the weak link is present at the middle or end of the chain which caused a greater energy waste.

Although this experimental study is limited in size, it provides two important conclusions. First, it does serve to confirm and validate our earlier findings from the analytical and simulation studies regarding the $\mathrm{PRR} \times$ distance metric. And second, it shows that the best reception metric is also a good metric for real deployments; we believe that the differences in energy savings and delivery rate with respect to the analytical and simulation sections are due to the size and density of the network.

\section{CONCLUSIONS AND FUTURE DIRECTIONS}

We have presented a detailed study of geographic routing in the context of lossy wireless sensor networks. Using a realistic link loss model, we have provided a mathematical analysis of the optimal forwarding distance for both ARQ and No-ARQ scenarios, as well as a detailed simulation study in which we proposed and evaluated several novel blacklisting and neighbor selection geographic forwarding strategies. We have also validated some of our approaches using real experiments on motes.

We have provided the first framework to study and analyze geographic forwarding strategies based on empirical packet loss models derived from real data measurements. Although, the results shown here are for a specific model; the framework, strategies and conclusions are quite robust and can be applied to other models as well. As a matter of 
fact, an earlier version of this paper used a channel model based on [9], which is less accurate than the current model. Even though the earlier model has a more uniform distribution of packet loss rates, the main results and conclusions observed are consistent between the two models.

Key results from our study indicate that the common greedy forwarding approach would result in very poor packet delivery rate. Efficient geographic forwarding strategies do take advantage of links in the high variance transitional region both for energy-efficiency and to minimize route disconnections. Also, reception-based forwarding strategies are generally more efficient than distance-based strategies; relative blacklisting schemes reduce disconnections and achieve higher delivery rates than absolute blacklisting schemes, but this could be at the cost of lower energy efficiency; and that ARQ schemes become more important as the network gets larger. Finally, an important forwarding metric that arose from our analysis, simulations and experiments, is $\mathrm{PRR} \times$ distance, particularly when ARQ is employed.

Among future extensions, we plan to study face (perimeter) routing in lossy networks. Face routing in a planar graph solves the dead-end problem after greedy forwarding fails. Interesting issues are expected on the interaction between lossy links and planarization. We also plan to consider the problem of inaccurate locations with the realistic link loss models. Further, we would like to take into account scenarios where the link losses vary with time, particularly in mobile networks where fading is a key concern. In such environments, blacklisting based on historic measurements may not be sufficient. Another possible extension is to consider the effect of MAC contention on our model and strategies. Finally, the mathematical model can be extended to provide directly the optimal forwarding distance from the $\mathrm{cm} f$ and $p d f$, without the need to provide the probability distribution of all distances as done in the analysis.

\section{REFERENCES}

[1] P. Bose, P. Morin, I. Stojmenovic and J. Urrutia. "Routing with Guaranteed Delivery in Ad Hoc Wireless Networks". Workshop on Discrete Algorithms and Methods for Mobile Computing and Communications (DialM 1999).

\begin{tabular}{|c|c|c|c|c|c|c|}
\hline$r(\%)$ & sce1 & sce2 & sce3 & sce4 & sce5 & sce6 \\
\hline GR & 0 & 2 & 32 & 0 & 0 & 94 \\
BR & 100 & 100 & 100 & 100 & 100 & 100 \\
PRR $\times$ DIST & 100 & 100 & 100 & 82 & 100 & 100 \\
\hline$t$ & sce1 & sce2 & sce3 & sce4 & sce5 & sce6 \\
\hline GR & 70 & 110 & 312 & 78 & 121 & 858 \\
BR & 652 & 407 & 730 & 754 & 701 & 903 \\
PRR $\times$ DIST & 563 & 425 & 632 & 547 & 560 & 883 \\
\hline Relative & & & & & & \\
$E_{\text {eff }}(\%)$ & sce1 & sce2 & sce3 & sce4 & sce5 & sce6 \\
\hline GR & inf & inf & +54 & inf & inf & +3 \\
BR & +16 & -4 & +16 & +14 & +25 & +2 \\
PRR $\times$ DIST & 0.0 & 0.0 & 0.0 & 0.0 & 0.0 & 0.0 \\
\hline
\end{tabular}

Table 1: Empirical Results for Different Forwarding Strategies. Notice that the relative $E_{\text {eff }}$ of PRR $\times$ DIST is 0.0 since it is used as the basis of comparison.
[2] G. G. Finn. "Routing and Addressing Problems in Large Metropolitan-Scale Internetworks". Tech. Rep. ISI/RR-8\%-180, ISI, Mar. 1987.

[3] B. Karp and H.T. Kung. "GPSR: Greedy Perimeter Stateless Routing for Wireless Networks". ACM MOBICOM 2000.

[4] E. Kranakis, H. Singh and J. Urrutia. "Compass Routing on Geometric Networks". In Proc. 11th Canadian Conference on Computational Geometry, August 1999.

[5] F. Kuhn, R. Wattenhofer and A. Zollinger. "Worst-Case Optimal and Average-Case Efficient Geometric Ad-Hoc Routing". ACM Mobihoc 2003.

[6] D. Kotz, C. Newport and C. Elliott. "The mistaken axioms of wireless-network research". Technical Report TR2003-467, Dept. of Computer Science, Dartmouth College, July 2003.

[7] D. Ganesan, B. Krishnamachari, A. Woo, D. Culler, D. Estrin and S. Wicker. "Complex Behavior at Scale: An Experimental Study of Low-Power Wireless Sensor Networks". UCLA CS Technical Report UCLA/CSD-TR 02-0013, 2002.

[8] D. S. J. De Couto, D. Aguayo, J. Bicket and R. Morris. "A High-Throughput Path Metric for Multi-Hop Wireless Routing". ACM MobiCom, September 2003.

[9] A. Woo, T. Tong and D. Culler. "Taming the Underlying Issues for Reliable Multhop Routing in Sensor Networks". ACM SenSys, November 2003.

[10] J. Zhao and R. Govindan. "Understanding Packet Delivery Performance in Dense Wireless Sensor Networks". ACM Sensys, November 2003.

[11] G. Zhou, T. He, S. Krishnamurthy and J. A. Stankovic. "Impact of Radio Irregularity on Wireless Sensor Networks". ACM MobiSys 2004.

[12] M. Mauve, J. Widmer and H. Hartenstein. "A Survey on Position-based Routing in Mobile Ad Hoc Networks". IEEE Network Magazine, Vol. 15, No. 6, pp. 30-39, November 2001.

[13] K. Seada and A. Helmy. "Geographic Protocols in Sensor Networks". USC Technical Report, July 2004.

[14] A. Cerpa, N. Busek and D. Estrin. "SCALE: A tool for Simple Connectivity Assessment in Lossy Environments". CENS Tech. Rep., September 2003.

[15] A. Cerpa, J. L. Wong, L. Kuang, M. Potkonjak and D. Estrin. "Statistical Model of Lossy Links in Wireless Sensor Networks". CENS Tech. Rep., April 2004.

[16] M. Zuniga and B. Krishnamachari, "Analyzing the Transitional Region in Low Power Wireless Links", IEEE Secon 2004.

[17] F. Silva, J. Heidemann and R. Govindan, Network Routing API 9.1, ISI Laboratory for Embedded Networked Sensor Experimentation, June 12th, 2003. Available online at http://www.isi.edu/ilense/pubs/

[18] T. S. Rappapport. "Wireless Communications: Principles and Practice". Prentice Hall. 